\title{
Vision Assisted Pick And Place Robotic ARM
}

\author{
Nisha ${ }^{1}$, Dinesh Kumar $^{2}$, Sekar $^{3}$ and Indira ${ }^{4}$ \\ ${ }^{1}$ University Science Instrumentation Centre, Madurai Kamaraj University, Madurai, \\ Tamilnadu, India \\ ${ }^{2}$ St. Joseph's College, Trichy, Tamilnadu, India \\ ${ }^{3}$ University Science Instrumentation Centre, Madurai Kamaraj University, Madurai, \\ Tamilnadu, India \\ ${ }^{4}$ University Science Instrumentation Centre, Madurai Kamaraj University, Madurai, \\ Tamilnadu, India
}

\begin{abstract}
This paper presents the design of a Vision assisted pick and place robotic Arm. The main objective of the paper is to pick and place an object from one place to other by 2 DOF robotic arm. USB camera is used as a vision sensor to measure the dimensions of the object to be picked. The USB camera collects the image of the object is transferred to the LabVIEW API with image processing toolkits and modules to process the image. The processed dimension of the object is transmitted via RS-232 serial communication to the microcontroller LPC2129. The appropriate PWM signal is generated by LPC2129 respectively to the servomotors. The robotic arm is designed with servomotors. Digital image processing algorithms are implemented to process the image captured by the USB camera to find the exact dimension of the object thereby to assist the robotic arm to finest. NI-IMAQ - Machine vision based functions are implemented and the results are presented.
\end{abstract}

\section{KEYWORDS}

Robotic Manipulator, NI-IMAQ-Machine Vision, Image Processing, LPC2129 microcontroller

\section{INTRODUCTION}

A robotic arm is a robotic manipulator, designed with different Degrees of Freedom depending upon the autonomous application where it is sorted. Now a day's several industrial automation are carried out with Robotic arm such as arc welding, automobile manufacturing industries makes use for painting and engine assembling [1]. The robotic arm to serve as a human in the industrial environment requires unique combination of embedded system architecture with its mechanical design. Among them the actuators implemented with the robotic arm designs are more consciously chosen. In general all motors fall under the category of actuators, the Servo motors are widely used with the construction of robotic arms [3]. The servo motors more appropriate to be used as its unique way of rotation characteristics. So, an entire mechatronic system would frame the robotic arm. The DOF of any robotic arm defines the ultimate efficiency equivalent to a human. Number of motors usage is dependant of the application for example in this proposed work the system is developed for a pick and place application [2]. Therefore the robotic arms are enabled with high torque motors to pick and move objects to new location. Three major parts of the robotic arm gripper, wrist and base are constructed with the servomotors [7]. The functioning of servomotors and working of robotic arm is discussed. The mechatronic design is completely capable of doing the defined job on the fields but it has to be monitored periodically to avoid some issues. The robotic arm is constructed with a microcontroller which thereby has provisions 
to interface with a PC [10]. The microcontrollers interfaced with the PC works dynamic in bidirectional way to get input from the PC and provide control signals as output to the servomotors which controls the robotic arm. On emergency situation the user can control the robotic arm from the PC. This is the usual case in the autonomous systems previously; this system design proposed is developed with vision sensor - USB camera to assist the robotic arm in absence of human and make the entire system as fully automated closed loop system [3]. The USB camera captures the image of the object to be picked and move to new location [9]. The captured image is further processed with Image processing algorithms which are running on PC with the NI-LabVIEW IMAQ Machine Vision tools and function modules [5][7]. LabVIEW IMAQ toolsets provide sophisticated functions to implement the Digital Image Processing algorithms in a PC [5]. In this work different digital image processing algorithms carried out to measure the exact dimensions of the object are presented.

\section{HARDWARE DESCRIPTION}

The main objective of this paper is to visually track the object and transmit the image to remote PC running LabVIEW software to find out the object dimensions and send the appropriate control signals to the Robotic arm via RS 232 serial communication to pick and place the object at expected location from unknown location. The system can be controlled by LabVIEW in PC.

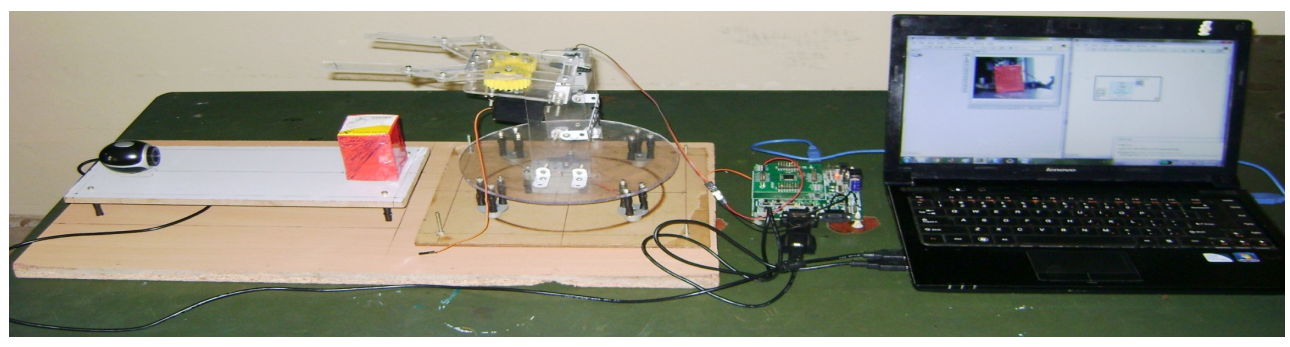

Figure 1. Overall view of Pick and place Robotic Arm assist by LabVIEW Machine Vision

\subsection{Robotic Arm}

The overall system of pick and place robotic arm was designed using acrylic sheets. Acrylic sheet is less weight, low cost and easy to cut. The design end effector is very simple. In gripper (end effector) design the standard wheels are used to give support to the whole mechanical assembly. Approximately the gripper weight is 100 grams. The one wheel has connected with servo motor shaft and another wheel has dummy wheel. The rotating the shaft dummy wheel is automatically rotated. The total system was controlled using three servo motors. The servo motor torque is $4 \mathrm{Kg} . \mathrm{cm}$. the maximum angle of the servo motor is 180 degrees.

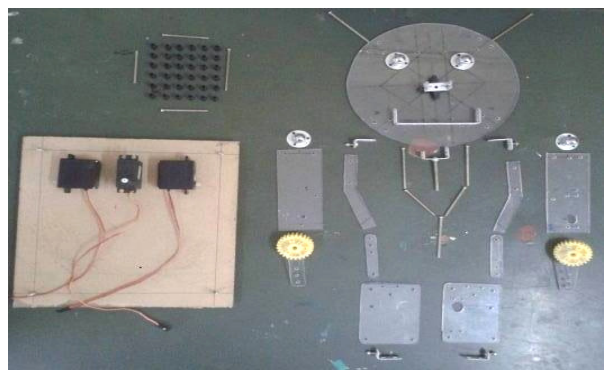

Figure 2. Components of Pick and place Robotic Arm 


\subsubsection{Base}

The figure 3 shows the base of the robotic arm. The four caster wheels are used to connect the round shape of the acrylic sheet. The base is connected to one servo motor and used to rotate the total system of arm. The length and width of the round shape acrylic sheet is $19.7 \mathrm{~cm}$ and $9.2 \mathrm{~cm}$.

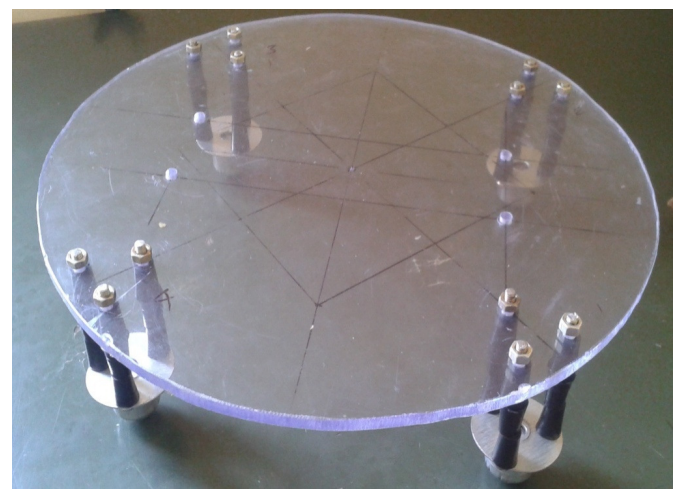

Figure 3. Base of Robotic Manipulator

\subsubsection{Wrist}

The figure 4 shows the wrist of the robotic arm. The wrist is robotic arm body to move freely up and down of the robotic arm. The wrist is connected to base and one servo motor. The servo motor torque is $5 \mathrm{~kg} . \mathrm{cm}$. The height of the wrist is $12.3 \mathrm{~cm}$ and width of the wrist is $4.0 \mathrm{~cm}$.

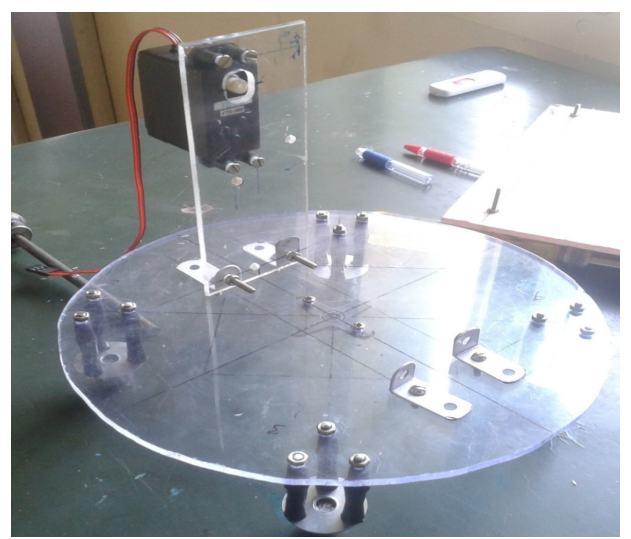

\subsubsection{Gripper}

Figure 4. Wrist of Robotic Manipulator

The figure 5 shows the gripper (end effector) of the robotic arm. The gripper is used to pick the object. The maximum gripper open is $9.3 \mathrm{~cm}$ for 180 degrees and close position is $1.0 \mathrm{~cm}$ for 0 degree. The gripper is connected one servo motor and used to pick and place the object. 


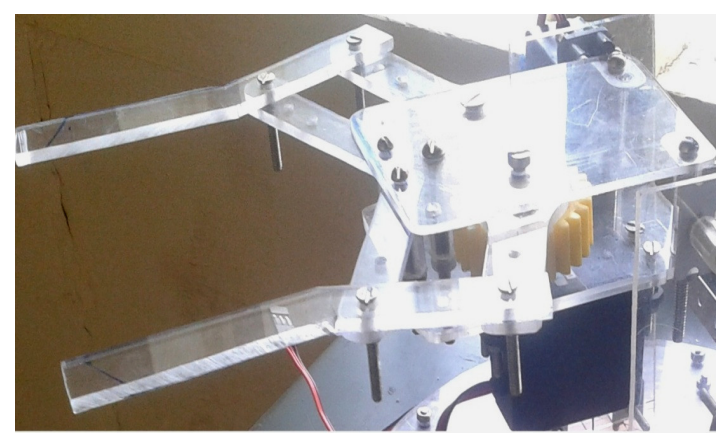

Figure 5. Gripper of Robotic Manipulator

\subsubsection{Design of Robotic Manipulator}

The complete system of robotic arm was designed using three servo motors. The servo motor is used to join the each link of the arm. The total weight of the system is 500 grams and total height is $19 \mathrm{~cm}$

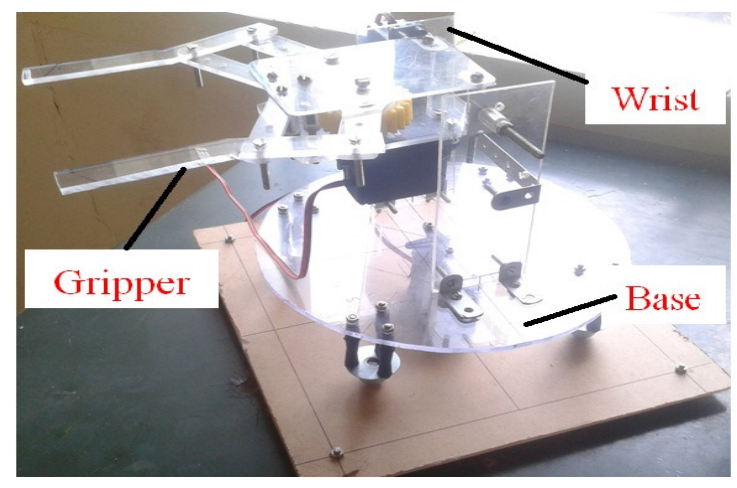

\subsection{Schematic Diagram}

Figure 6. Robotic Manipulator

The figure 7 shows the complete schematic diagram of robotic arm. The full system was controlled by using servo motors. The servo motors are used in robotics. The servo motor is three wires and the control signal of servo motor is connected to the microcontroller pins of LPC2129. The microcontrollers are $6 \mathrm{PWM}$ outputs and supply voltage is $3.3 \mathrm{~V}$. The camera is connected to $\mathrm{PC}$ and MAX $232 \mathrm{IC}$ is connected to the Microcontroller. 


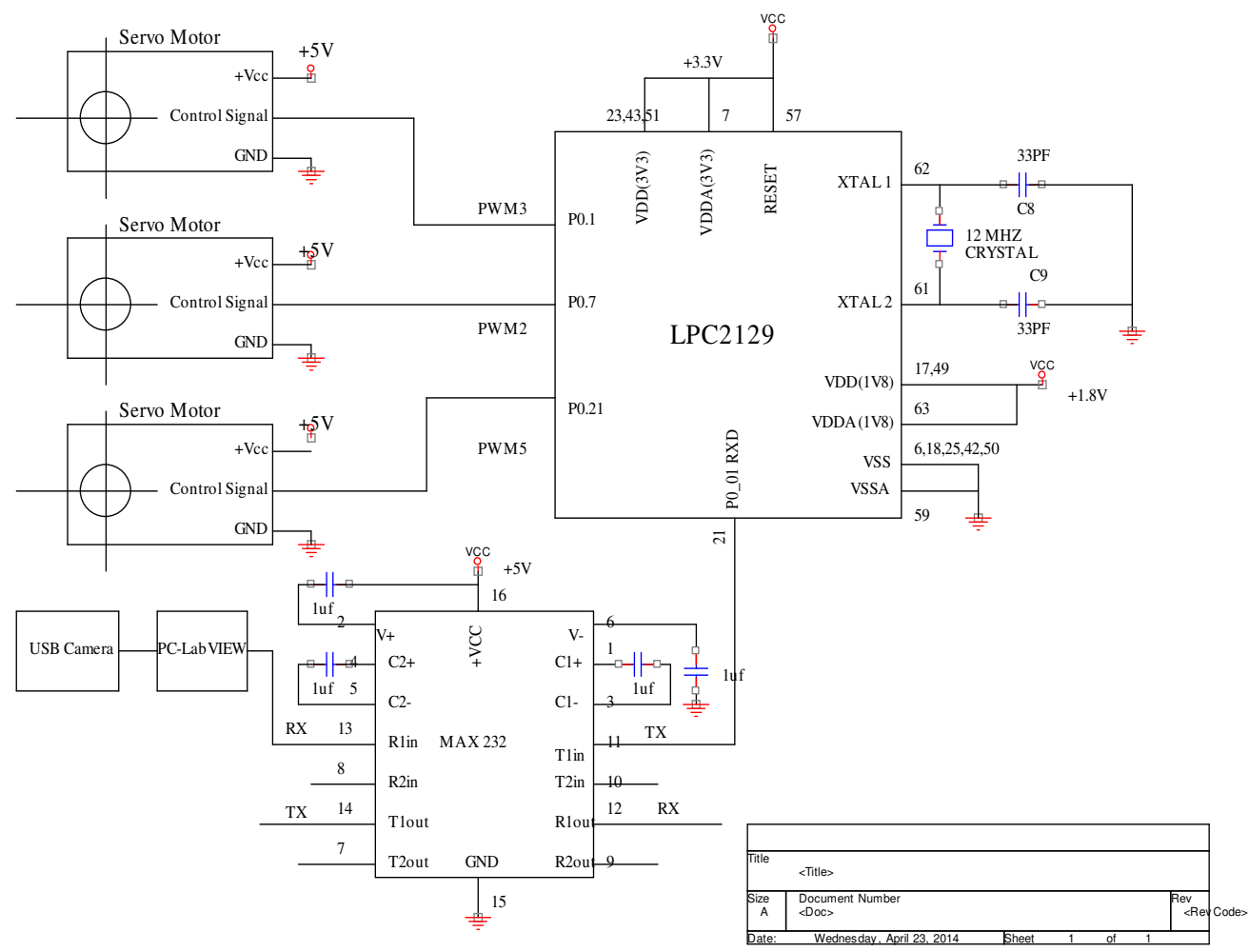

Figure 7. Schematic diagram

\section{SOFTWARE DESCRIPTION}

\subsection{LabVIEW Machine Vision}

Machine vision is the technology widely used in industry and research [8]. NI Vision supports low-cost USB camera and Wireless webcams. NI offers three distinct software packages to the needs of programmers, which include world-class image processing algorithms and image acquisition functions. [5]

- Vision Development Module is a LabVIEW Add-On that installs the entire sub VIs and functions needed to acquire images from a camera and do analysis on them.[5]

- Vision Builder for Automated Inspection is a separate application that used to configuration based interface.[5]

- The third component of NI Vision Software is the Vision Acquisition Software- a set of hardware drivers (including IMAQ $\mathrm{dx}$ ) that allows NI software to communicate with camera hardware.[5]

\subsection{Algorithm for LabVIEW programming}

The following basic steps that are used to Creating NI IMAQ Vision Application program [5][7][8]: 
$>$ Setup imaging system

$>$ Calibrate imaging system

$>$ Acquire or Read an image:

$>$ Display an image:

$>$ Attach Calibration information:

$>$ Analyze an image:

$>$ Improve an image:

\subsection{Image Acquisition}

First acquire the image from camera using vision acquisition and select the acquisition type camera setting and indicator. In front panel to display the image, image type and image coordinates.

\subsection{Image Processing- Pattern Matching Algorithm}

\subsubsection{Step 1: RGB to Grayscale conversion}

The display image is processed using image processing tools. First image is converted into RGB to grayscale using IMAQ Mask image. Then the image processed using vision assistant. In color pattern matching tool the input image is selected and save it template for template image. The input image and template image are matched then image $\mathrm{x}$, y position displayed in pixels. The pixel value is converted into length using the below formula

$$
1 \text { pixel }=0.026458333 \mathrm{~cm}
$$

In pattern matching algorithm $\mathrm{x}, \mathrm{y}$ results are $4.49 \mathrm{~cm}$ and $3.73 \mathrm{~cm}$. in real world object $\mathrm{x}, \mathrm{y}$ results are $6.5 \mathrm{~cm}$ and $5 \mathrm{~cm}$.

\subsubsection{Step 2: Pattern matching algorithm}

In this method to find object dimension using pattern matching algorithm. Pattern Matching is used to searches for a pattern, or template image, in an inspection image. The template image is saved in the disk. To check whether the input image and template image matched or not. If it is matched number of matches is one.

\subsubsection{Step 2: Edge Detection algorithm}

In this method to find object dimension using edge detection. There are different types of methods are used in edge detection sobel, prewitt, sigma and differentiation. In this algorithm prewitt method is used to display an object edges. Then select the edge of object using IMAQ rectangular and applied to the ROI descriptor to find the object $\mathrm{x}$ and $\mathrm{y}$ pixels value

\section{TEST AND ANALYSIS}

\subsection{Read an image}

This is simple program for acquire and display image using USB camera. The figure 8 and figure 9 shows the VI front panel and block diagram of acquire and display an image. 
Advances in Vision Computing: An International Journal (AVC) Vol.2, No.3, September 2015

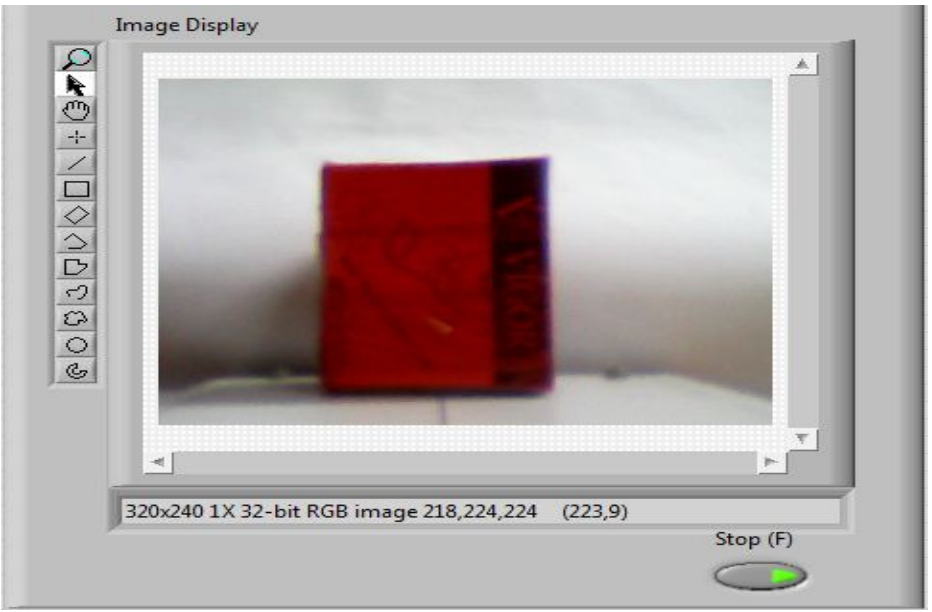

Figure 8. Front Panel of Image acquisition

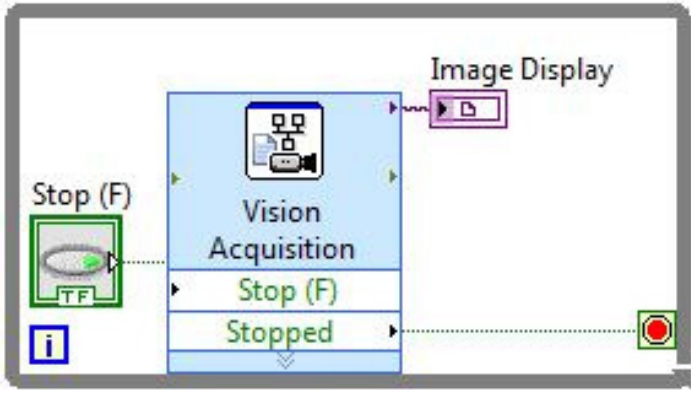

Figure 9. Block diagram of Image acquisition

\subsection{Pattern matching algorithm}

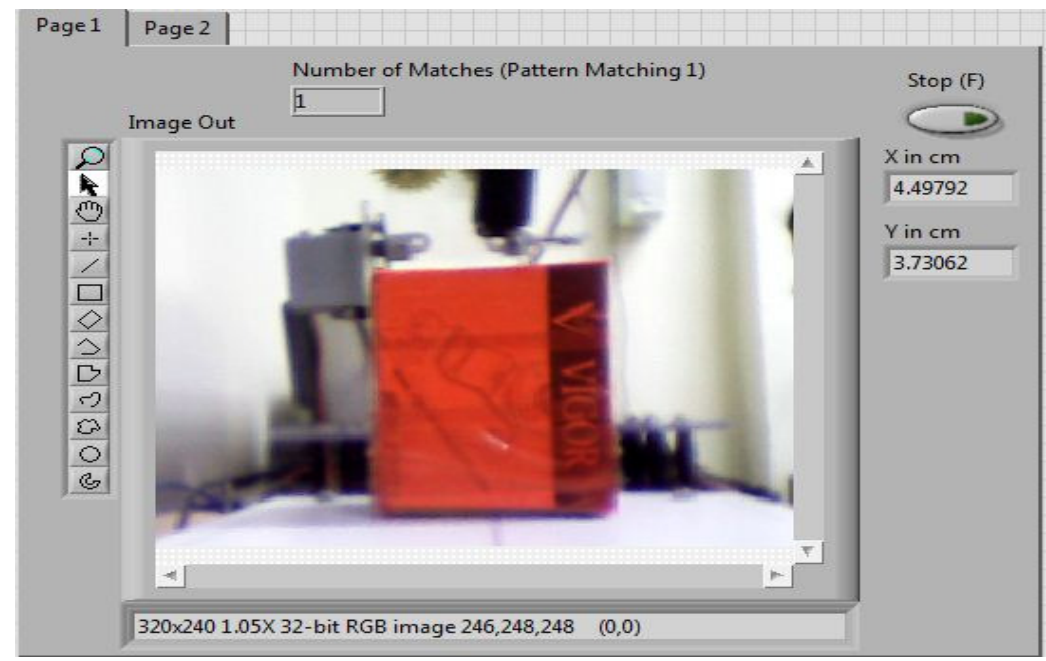

Figure 10. Front Panel of Image Processing- Pattern matching 
Advances in Vision Computing: An International Journal (AVC) Vol.2, No.3, September 2015

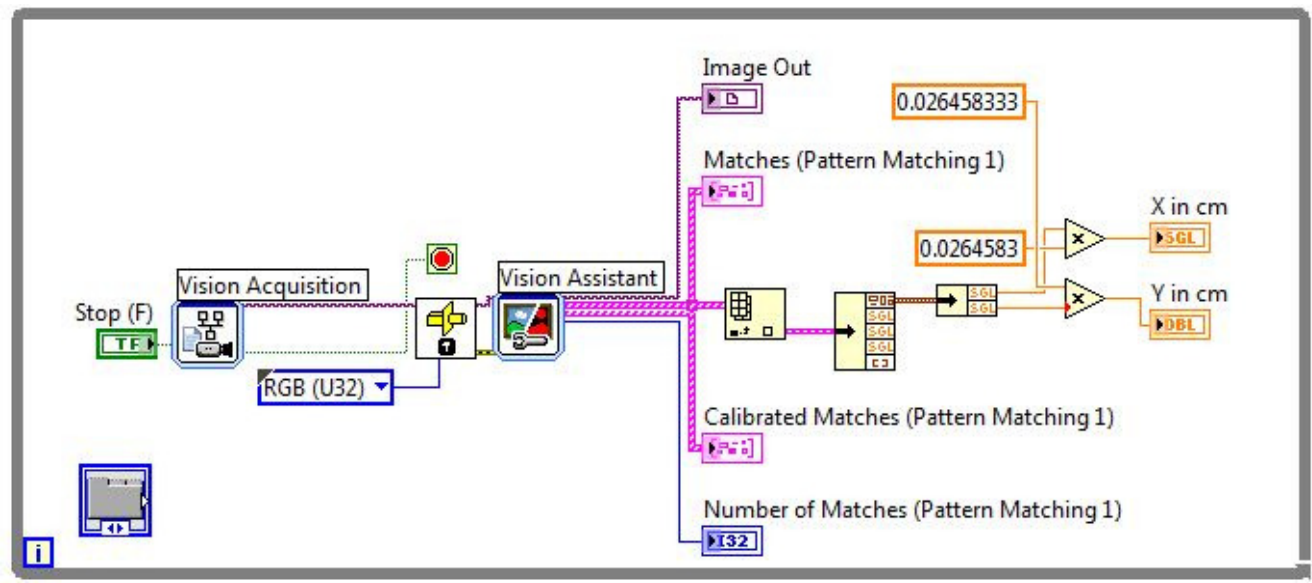

Figure 11. Block diagram of Image Processing- Pattern matching

\subsection{Edge detection algorithm}

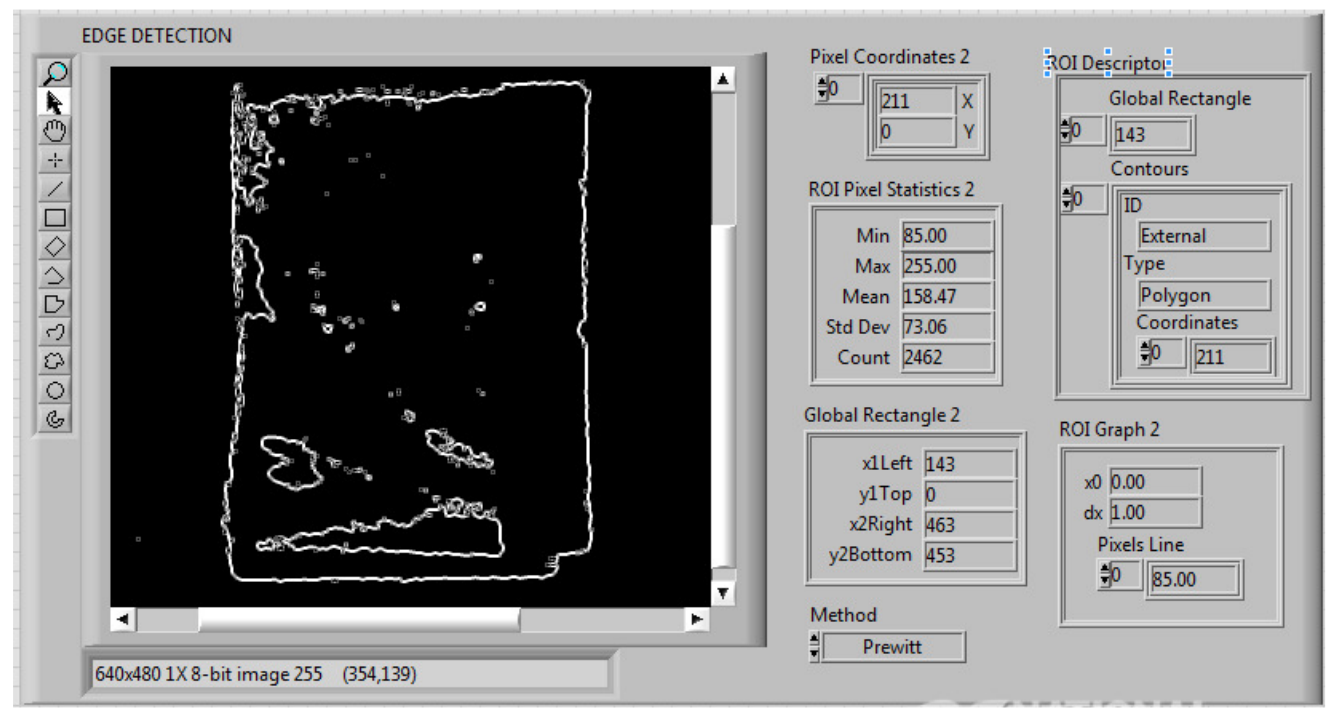

Figure 12. Front Panel of Image Processing- Edge detection 


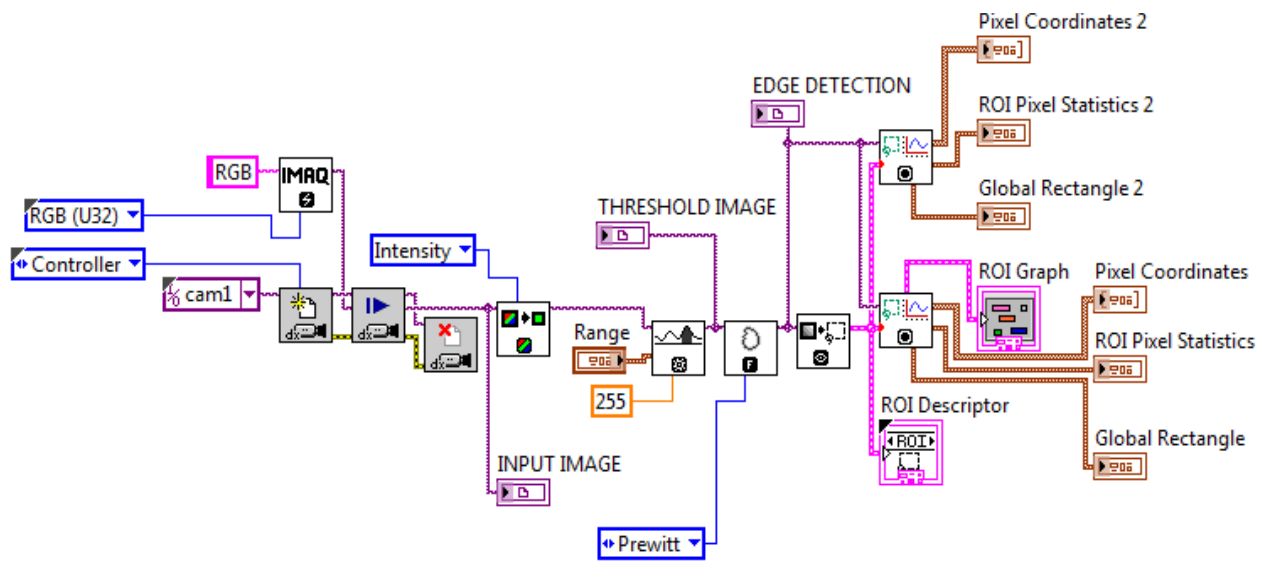

Figure 13. Block diagram of Image Processing- Edge detection

Table 1. Test and Measurements.

\begin{tabular}{|l|l|l|l|}
\hline Sl. No & Trial & $\begin{array}{l}\text { Original object dimension } \\
\text { (in cm) }\end{array}$ & $\begin{array}{l}\text { Object dimension by NI } \\
\text { IMAQ (in cm) }\end{array}$ \\
\hline 1 & Object 1 & $\mathrm{X}=6.5 \mathrm{y}=5$ & $\mathrm{X}=4.49, \mathrm{y}=3.73$ \\
\hline 2 & Object 2 & $\mathrm{X}=7 \mathrm{y}=4$ & $\mathrm{X}=5.3 \mathrm{y}=2.9$ \\
\hline 3 & Object 3 & $\mathrm{X}=6 \mathrm{y}=4$ & $\mathrm{X}=4.6 \mathrm{y}=2.8$ \\
\hline
\end{tabular}

\section{CONCLUSIONS}

Nowadays, the use of robotic arm in several logistics for moving packages from one end to other end on some industries for fault detection applications with complex designs. In this work a USB camera based Vision Assisted Robotic Arm system is successfully constructed for pick and place an object from one place to other by of 2 DOF from the commercially available parts. The digital image processing algorithms are implemented on the practical design with the LabVIEW Machine Vision toolsets. In this system, the USB camera is used as a sensor captures the image of the object and the dimensions of the object are measured after processing the image in LabVIEW API. The object dimensions are determined by the edge detection and pattern matching algorithm using IMAQ Vision software tools. From the real-time test and measurement it is observed that the robotic arm requires a 7-DOF for a full-fledged working in pick and place applications. So, the work can be further carried out by increasing the use of servomotors in the body of robotic arm and making use of a high resolution 3D camera

\section{REFERENCES}

[1] Vishnu R.kale, V.A. kulkarani, (2013) “Object Sorting System Using Robotic Arm” International Journal of Advanced Research in Electrical, Electronics and Instrumentation Engineering, Vol. 2, Issue 7.

[2] Shyam.R.Nair, (2012) "Design of an Optically Controlled Robotic Arm for Picking and Placing an Object”, International Journal for Scientific and Research Publications, Vol 2, Issue 4.

[3] Bikarna pokharel, (2013) "Machine Vision and Object Sorting" Automotive Engineering.

[4] Mohd Ashiq Kamaril Yusoff, Reza Ezuan Samin, Babul Salam Kader Ibrahim, (2012) "Wireless Mobile Robotic Arm", International Symposium on Robotics and Intelligent Sensors. 
Advances in Vision Computing: An International Journal (AVC) Vol.2, No.3, September 2015

[5] National Instruments, "IMAQ Vision for LabVIEW user manual"

[6] Waquar Mazhar (2013), "Haptics Based Gesture Controlled Robot" International Journal of Engineering and Technology, $\operatorname{Vol}(6)$

[7] Vivek Bhojak, Girish Kumar Solanki, Sonu Daultani June(2015), "Gesture Controlled Mobile Robotic Arm Using Accelerometer", International Journal of Innovative Research in Science Engineering and Technology, Vol(4), Issue 6.

[8] Ruben Posada-Gomez, Oscar Osvaldo Sandoval-Gonzalez, Albino Martinez Sibaja, Otneil and Giner, "Digital Image Processing Using LabView", www.intechopen.com.

[9] Shahed Shojaeipour, Sallehuddin Mohamed Haris, Elham Gholami and Ali Shojaeipour, "Webcambased Mobile Robot Path Planning using Voronoi Diagrams and Image Processing", International Conference on Applications of Electrical Engineering.

[10 ]NXP B. V. (founded by Philips) (2008). "UM10114 LPC21xx and LPC22xx User Manual”, Rev. 032 April 2008. 